\title{
Validación de la Entrevista de Carga de Zarit en cuidadores primarios informales de pacientes con diagnóstico de enfermedades mentales ${ }^{1}$
}

\section{Validation of the Zarit Burden Interview in informal primary caregivers of patients with diagnosis of mental disorders}

\author{
Michelle Flores-Terrones ${ }^{2}$, Óscar Galindo-Vázquez ${ }^{3-4}$, \\ Janet Jiménez-Genchi ${ }^{5}$, Liliana Rivera-Fong ${ }^{l}$ y Eva González-Rodríguez ${ }^{l}$
}

Citación: Flores T., M., Galindo V., O., Jiménez G., J., Rivera F., L. y González R., E. (2019). Validación de la Entrevista de Carga de Zarit en cuidadores primarios informales de pacientes con diagnóstico de enfermedades mentales. Psicología y Salud, 29(1), 17-24.

\section{RESUMEN}

\begin{abstract}
Introducción: Un trastorno mental es un síndrome caracterizado por una alteración del estado cognitivo, la regulación o el desarrollo del individuo. Este grupo de pacientes puede requerir un cuidador primario informal (CPI en lo sucesivo) que lo asista en sus necesidades físicas, emocionales, sociales, de atención médica o cohesión familiar. En este contexto, la Entrevista de Carga de Zarit (ECZ ) es un instrumento utilizado en la evaluación de dichos CPI. Objetivo: Validar la ECZ en una muestra de CPI de pacientes con diagnostico psiquiátrico. Método: Se empleó un diseño transversal, con un muestreo no probabilístico. Participaron 235 CPI, quienes contestaron concurrentemente la ECZ y el cuestionario SF-36. Resultados y Discusión: La versión obtenida en el presente estudio mostró un instrumento de 17 ítems con una consistencia interna satisfactoria de la escala global. Se obtuvieron tres subescalas: Impacto del cuidador, Relaciones interpersonales y Expectativas de autoeficacia, así como un indicador cognitivo. La validez concurrente mostró resultados significativos. El análisis psicométrico realizado indica que la ECZ en CPI de pacientes con diagnóstico psiquiátrico tiene una buena consistencia interna, mayor que la de la versión original. Comparada con las demás validaciones del Zarit, mostró una estructura factorial similar. La relevancia de los resultados obtenidos radica en su utilidad clínica y de investigación en grupos de cuidadores que pueden requerir atención psicosocial.
\end{abstract}

Palabras clave: Cuidadores primarios informales; Pacientes; Psiquiatría; Salud mental; Sobrecarga.

\begin{abstract}
Introduction: A mental disorder is a syndrome characterized by clinically significant impairment of cognitive, regulatory or behavioral status of an individual. These patients may require informal primary caretakers (IPC) to assist them in physical, emotional, social, health care and / or family cohesion. In this context, the Zarit Burden Interview (ZBI) is an instrument used in the evaluation of IPCS. Objective: To validate the ZBI in a sample of informal primary caregivers of patients with psychiatric diagnoses. Method: A cross-sectional design was used, with a non-probability sampling. A total of 235 informal primary caregivers of patients with psychiatric diagnosis participated, they concurrently answered the ZBI and the SF-36 health questionnaire. Results: the version used in this
\end{abstract}

\footnotetext{
${ }^{1}$ Los autores agradecen a Lundbeck por su apoyo en la impresión de los instrumentos para esta investigación. Artículo recibido el 2 de abril y aceptado el 2 de julio de 2018 .

${ }^{2}$ Universidad Latina, Campus Sur, Pedro Henríquez Ureña 173, Col. Los Reyes, Del. Coyoacán, Ciudad de México, México.

${ }^{3}$ Servicio de Psicooncología del Instituto Nacional de Cancerología, Av. San Fernando 22, Col. Sección XVI, Del. Tlalpan, 14080 Ciudad de México, México, tel. (55)56-28-04-00, ext.60259, correo electrónico: psigalindo@yahoo.com.mx.

${ }^{4}$ Facultad de Psicología, Universidad Nacional Autónoma de México, Av. Universidad 3004, Col. Copilco Universidad, Del. Coyoacán, 04510 Ciudad de México, México.

${ }^{5}$ Hospital Psiquiátrico “Fray Bernardino Álvarez”. San Buena Aventura 2, Del. Tlalpan, 14000 Ciudad de México, México.
} 
study revealed a successful 17-item instrument regarding internal consistency of the global scale. Three subscales were obtained: Caregiver impact, Interpersonal relationships, and Self-efficacy expectations, as well as a cognitive indicator. The concurrent validity showed significant results. Discussion: The psychometric analysis performed in this research indicates that the ZBI of patients with psychiatric diagnosis has an internal consistency higher than the original version. Compared with other Zarit validations, and it has a similar factorial structure. The relevance of these results lies in its clinical and research usefulness in a group of IPC who may require psychosocial care.

Key words: Informal primary caregivers; $\mathrm{Pa}$ tients; Psychiatry; Mental health; Overload.

\section{INTRODUCCIÓN}

$\mathrm{U}$ n trastorno mental es un síndrome caracterizado por una alteración clínica significativa del estado cognitivo, la regulación emocional o el comportamiento del individuo, misma que refleja una disfunción de los procesos psicológicos, biológicos o del desarrollo que subyacen a su función mental (American Psychatric Association, 2014).

La Organización Mundial de Salud señaló en 2004 que la esquizofrenia, la depresión, la epilepsia, la demencia y los trastornos neurológicos y por uso de sustancias representaban 13\% de la carga mundial de las enfermedades discapacitantes, superando así a las enfermedades cardiovasculares y al cáncer (cf. también Secretaría de Salud, 2006).

Hasta las últimas décadas del siglo pasado se subestimaba el impacto de los trastornos mentales, entre los cuales la depresión (de 4.6 a 4.8\%), la fobia social (de 5.4 a $5.8 \%$ ) y el trastorno por estrés postraumático (de 5.6 a $5.4 \%$ ) son los que producen los mayores niveles de discapacidad (Lara, Medina-Mora, Borges y Zambrano, 2007).

Los trastornos mentales ocupan un importante lugar en la carga global de la enfermedad en el mundo y aumentaron de $5.4 \%$ en 1990 a $7.4 \%$ en 2010 (Murray et al., 2012).

Por ejemplo, la depresión es una enfermedad que tiene un impacto significativo en la calidad de vida y en el funcionamiento cotidiano de la persona. Se observa que sólo una proporción pequeña de quienes se ven afectados acuden a tratamiento, y quienes lo hacen han demorado mucho tiempo su decisión de hacerlo; aunado a esto, únicamente la mitad recibe un tratamiento mínimo adecuado (Berenzon, Lara, Robles y Medina-Mora, 2013).

Este grupo de pacientes puede llegar a requerir atención en labores de higiene, administración de medicamentos, transporte a los centros hospitalarios, coordinación de citas médicas, atención emocional y comunicación con el equipo de salud, por lo que sus CPI desempeñan un importante papel en la atención domiciliaria y hospitalaria de los pacientes (Astudillo, Martínez, Muñoz, Pacheco y Sepúlveda, 2012; Islas, Ramos, Aguilar y García, 2006).

La atención que el CPI da al paciente aumenta conforme progresa la discapacidad de la enfermedad (Camacho, Yokehed y Jiménez, 2010). El CPI extiende progresivamente el tiempo de cuidado de la persona, disminuye su propio tiempo de descanso, y sobreprotege a aquélla, lo que implica un mayor tiempo, trabajo y dedicación, haciendo más probables los riesgos asociados al estrés y el agotamiento (Aguilar, 2014). Esto ha llevado analizar la carga de los CPI, que suele estar provocada por la dificultad que conlleva estar a cargo de una persona enferma, el cambio radical de su modo de vida y el desgaste que provoca ver como un ser querido pierde progresivamente sus facultades físicas y psíquicas. En la literatura se describe el "síndrome del cuidador primario informal" como el fenómeno relacionado directamente con la sobrecarga física y emocional (Kessler et al., 2007).

El CPI es "la persona del entorno de un paciente que asume voluntariamente el papel de responsable para el cuidado en un amplio sentido. Este individuo está dispuesto a tomar decisiones por el paciente y a cubrir las necesidades básicas del mismo, ya sea de manera directa o indirecta" (Vázquez et al., 2015).

Entre las principales afectaciones en el CPI de pacientes con diagnostico psiquiátrico están las emocionales (irritabilidad, angustia, enojo), de 25 a $72 \%$; problemas económicos en $58 \%$; problemas físicos en $44 \%$, afectaciones sociales, como falta de convivencia con amigos, vecinos, pareja o familia, y disminución en actividades recreativas en 33\%. Los CPI pueden desarrollar trastornos mentales como la depresión, más aún en el 
género femenino, con nivel socioeconómico bajo, con menor nivel educativo y estrategias de afrontamiento insuficientes (Astudillo et al., 2012).

De acuerdo con Alpuche, Ramos, Rojas y Figueroa (2008), el síndrome de sobrecarga que presentan los CPI presenta dos dimensiones: la carga objetiva, que implica la atención a un paciente, la dedicación al desempeño del rol del cuidado, el aumento de las demandas de cuidado, el tiempo dedicado, la carga física y la exposición a situaciones estresantes, mientras que la carga subjetiva se ha definido como las actividades y relaciones emocionales ante la experiencia del cuidado, así como la percepción de la situación del cuidado.

En este contexto, la Entrevista de Carga de Zarit (ECZ en lo sucesivo) (Zarit, Reever y Bach-Peterson, 1980) para CPI de pacientes con enfermedades crónico-degenerativas es un instrumento sumamente utilizado para identificar la sobrecarga en CPI. Aun así, se carece de datos sobre la validez y confiabilidad en CPI de pacientes con diagnóstico psiquiátrico, por lo que el objetivo de este estudio fue obtener las propiedades psicométricas del instrumento en ese grupo de CPI.

\section{MÉTODO}

\section{Participantes}

Participaron 235 CPI de ambos sexos, con un promedio de 51.40 años de edad, que atendían a pacientes con diagnóstico psiquiátrico (Tabla 1). La muestra se obtuvo por disponibilidad en el Hospital Psiquiátrico "Fray Bernandino Álvarez", ubicado en la Ciudad de México.

Tabla 1. Descripción de la muestra de cuidadores primarios informales (CPI).

\begin{tabular}{|c|c|c|c|c|c|}
\hline Características & Frec. & $\%$ & Diagnóstico & Frec. & $\%$ \\
\hline Número & 235 & 100.0 & Esquizofrenia y otros trastornos psiquiátricos & 145 & 61.7 \\
\hline \multicolumn{3}{|l|}{ Sexo } & Trastornos del estado de ánimo & 27 & 11.4 \\
\hline Masculino & 59 & 25.1 & Trastornos de personalidad & 12 & 5.1 \\
\hline Femenino & 176 & 74.9 & Trastornos neurodegenerativos & 16 & 6.8 \\
\hline \multicolumn{3}{|l|}{ Escolaridad } & Trastornos obsesivo-compulsivos & 1 & 0.4 \\
\hline Ninguna & 14 & 6.0 & Trastornos orgánicos afectivos & 1 & 0.4 \\
\hline Primaria & 55 & 23.4 & Trastornos del desarrollo & 6 & 2.6 \\
\hline Secundaria & 66 & 28.1 & No se sabe & 27 & 11.5 \\
\hline Bachillerato & 54 & 23.0 & \multicolumn{3}{|l|}{ Tratamiento del paciente } \\
\hline Licenciatura & 39 & 16.6 & Fármacos & 229 & 97.4 \\
\hline Posgrado & 7 & 3.0 & Terapia electroconvulsiva (TEC) & 1 & 0.4 \\
\hline \multicolumn{3}{|l|}{ Estado civil } & Fármacos más TEC & 5 & 2.1 \\
\hline Solteros & 40 & 17.0 & \multicolumn{3}{|l|}{ Tiempo de diagnóstico } \\
\hline Casados o en unión libre & 97 & 41.3 & $0-5$ años & 138 & 61.7 \\
\hline Separados o divorciados & 48 & 20.4 & 6-10 años & 19 & 8.1 \\
\hline Viudos & 30 & 12.8 & $11-15$ años & 24 & 10.2 \\
\hline Otro & 20 & 8.5 & 16-20 años & 20 & 8.5 \\
\hline \multicolumn{3}{|l|}{ Relación con el paciente } & $>20$ años & 27 & 11.5 \\
\hline Hermano & 35 & 14.9 & \multicolumn{3}{|l|}{ Tiempo a cargo del paciente } \\
\hline Esposo(a) & 22 & 9.4 & $0-5$ años & 141 & 59.9 \\
\hline Padre o madre & 150 & 63.8 & 6-10 años & 24 & 10.2 \\
\hline Amigo(a) & 3 & 1.3 & $11-15$ años & 22 & 9.4 \\
\hline Hijo(a) & 18 & 7.7 & 16-20 años & 18 & 7.7 \\
\hline Sobrinos, tíos o abuelos & 7 & 3.0 & $>21$ años & 24 & 10.2 \\
\hline & & & $>31$ años & 5 & 2.1 \\
\hline & & & $>41$ años & 1 & 0.4 \\
\hline
\end{tabular}




\section{Procedimiento}

Durante las visitas que hacían los CPI antes o después de la consulta de su paciente en los servicios ambulatorios o en hospitalización, se les solicitó su participación, para lo cual debían firmar una carta dando su consentimiento informado para colaborar en el estudio bajo los lineamientos de la investigación aprobados por los Comités de Investigación y de Ética del citado hospital.

Los criterios de inclusión fueron, a saber: ser mayor de edad, CPI de un paciente con diagnóstico psiquiátrico, aceptar voluntariamente participar en el estudio y saber leer y escribir. Los criterios de exclusión establecidos fueron los de sufrir afectaciones visuales o auditivas severas y tener conocimientos y preparación profesional para atender a una persona enferma. El único criterio de eliminación fue que el CPI decidiera no continuar en la investigación.

\section{Instrumentos}

\section{Cedula de identificación.}

Se incluyó una cédula de identificación del participante en la que se recabaron los siguientes datos: edad, sexo, nivel de estudios, estado civil, condición física, relación con el paciente, tiempo que tenían a su cargo el paciente, tiempo transcurrido desde el diagnóstico del paciente, y diagnóstico, edad y sexo del mismo.

Entrevista de Carga de Zarit para Cuidadores Primarios (ECZ) (Zarit et al., 1980).

La ECZ es un cuestionario autoadministrado que consta de 22 ítems y tres subescalas (Impacto de la atención, Relación interpersonal y Expectativas para la autoeficacia). Con un coeficiente de confiabilidad alfa de Cronbach de 0.88, es un instrumento que ha sido validado en cuidadores de población geriátrica y de pacientes con alzheimer, donde alcanza un coeficiente alfa de entre .83 y .91. Mide la carga percibida por el cuidador a través de una escala Likert que va de 0 (nunca) a 4 (siempre). Sumando los 22 reactivos se obtiene un índice único de la carga, con un rango de puntuación de 0-88. En otras poblaciones de CPI, ha mostrado coeficientes alfa de Cronbach de .83 a .91 .
Es un instrumento continuamente usado en investigaciones sobre CPI de pacientes con enfermedades crónico-degenerativas en todo el mundo (Alpuche et al., 2008; Galindo et al., 2015; Montero et al., 2014; Zarit et al., 1980; Zarit, Orr y Zarit, 1985).

\section{Cuestionario del Estado de Salud (SF-36)}

(Ware, 2000).

El SF-36 detecta tanto estados positivos de salud como negativos, salud física y salud mental. Consta de 36 ítems que exploran ocho dimensiones del estado de salud: función física, función social, limitaciones del rol, problemas físicos, problemas emocionales, salud mental, vitalidad, dolor y percepción de la salud general.

\section{Análisis}

Para el análisis de los datos se usó el paquete estadístico SPSS, versión 20, de Windows. Se determinó la $t$ de Student para muestras independientes a fin de comparar los grupos; se determinó la estructura factorial por medio de un análisis factorial de componentes principales con rotación varimax. La relación entre la ECZ y el SF-36 se analizó mediante el coeficiente de correlación de Pearson, $y$ con el coeficiente alfa de Cronbach la consistencia interna.

\section{RESULTADOS}

\section{Estructura factorial}

Se realizó el análisis de distribución de frecuencias y se determinó el sesgo de la prueba en esa población; a la vez, se identificaron los cuartiles por extremos y se creó la nueva variable para grupos extremos, hecho lo cual se calculó la t de Student para muestras independientes con $p<0.05$; se calculó asimismo la consistencia interna y el análisis factorial exploratorio (método de componentes principales y rotación varimax), terminando con 17 reactivos que arrojaron un modelo con tres factores y un indicador con valores arriba de 1.00. El índice de adecuación muestral KMO fue significativo: 0.893 (Tabla 2). 
Tabla 2. Análisis factorial de la validación del ECZ para cuidadores primarios informales.

\begin{tabular}{|c|c|c|c|}
\hline $\begin{array}{l}\text { Escala global } \alpha=0.89 \\
\text { Varianza explicada }=55.7 \%\end{array}$ & $\begin{array}{c}\text { Carga } \\
\text { factorial }\end{array}$ & $\begin{array}{l}\text { Varianza } \\
\text { explicada }\end{array}$ & $\begin{array}{c}\text { Alfa } \\
\text { de Cronbach }\end{array}$ \\
\hline Factor 1: Impacto del cuidado & & \multirow{10}{*}{$33.4 \%$} & \multirow{10}{*}{0.863} \\
\hline 1. ¿Cree que su familiar le pide más ayuda de la que realmente necesita? & 0.521 & & \\
\hline $\begin{array}{l}\text { 2. ¿Cree que debido al tiempo que dedica a su familiar ya no dispone de tiempo } \\
\text { suficiente para usted? }\end{array}$ & 0.621 & & \\
\hline $\begin{array}{l}\text { 3. ¿Se siente agobiado por cuidar a su familiar y tratar de cumplir } \\
\text { otras responsabilidades en su trabajo o con su familia? }\end{array}$ & 0.657 & & \\
\hline 10. ¿Cree que su salud se ha deteriorado por cuidar a su familiar? & 0.780 & & \\
\hline 11. ¿Cree que no tiene tanta intimidad como le gustaría debido a su familiar? & 0.618 & & \\
\hline 12. ¿Cree que su vida social se ha resentido por cuidar a su familiar? & 0.622 & & \\
\hline 17. ¿Siente que ha perdido el control de su vida desde la enfermedad de su familiar? & 0.720 & & \\
\hline 19. ¿Se siente indeciso sobre qué hacer con su familiar? & 0.421 & & \\
\hline 22. ¿Se ha sentido sobrecargado por el hecho de cuidar a su familiar? & 0.720 & & \\
\hline \multicolumn{2}{|l|}{ Factor 2: Relación interpersonal } & \multirow{4}{*}{$10.3 \%$} & \multirow{4}{*}{0.609} \\
\hline 4. ¿Se siente avergonzado por la conducta de su familiar? & 0.772 & & \\
\hline $\begin{array}{l}\text { 6. ¿Piensa que su familiar afecta negativamente su relación con otros miembros } \\
\text { de la familia? }\end{array}$ & 0.627 & & \\
\hline 18. ¿Desearía dejar el cuidado de su familiar a otros? & 0.679 & & \\
\hline \multicolumn{2}{|l|}{ Factor 3: Expectativas de autoeficacia } & \multirow{4}{*}{$7.37 \%$} & \multirow{4}{*}{0.659} \\
\hline 8. ¿Cree que su familiar depende de usted? & 0.825 & & \\
\hline $\begin{array}{l}\text { 14. ¿Cree que su familiar parece esperar que usted sea quien lo cuide como si fuera } \\
\text { la única persona de quien depende? }\end{array}$ & 0.681 & & \\
\hline $\begin{array}{l}\text { 15. ¿Cree que no tiene suficiente dinero para cuidar a su familiar, además } \\
\text { de sus otros gastos? }\end{array}$ & 0.584 & & \\
\hline \multicolumn{2}{|l|}{ Indicador cognitivo } & \multirow{3}{*}{$6.35 \%$} & \multirow{3}{*}{0.733} \\
\hline 20. ¿Cree que debería hacer más por su familiar? & 0.877 & & \\
\hline 21. ¿Cree que podría cuidar mejor a su familiar? & 0.821 & & \\
\hline
\end{tabular}

\section{Consistencia interna}

La consistencia interna de la escala global mostró un índice adecuado $(\alpha=0.89)$. Se identificó una estructura de tres factores: impacto del cuidado, relaciones interpersonales y expectativas de autoeficacia, así como un indicador cognitivo, los cuales tuvieron valores $\alpha$ de .86 a .60, que explican $55.7 \%$ de la varianza. En términos generales, la consistencia puede considerarse adecuada.

\section{Validez externa por medio de correlaciones con medidas concurrentes}

Se identificaron correlaciones positivas y estadísticamente significativas ( $\mathrm{r}$ de Pearson). Fue el rol emocional el que correlacionó con todos los factores de la ECZ y la función física, el rol físico, y el dolor corporal con la mayoría de ellos, obteniéndose una $p<0.05$ (Tabla 3 ).

Así, la ECZ de cuidadores primarios informales de pacientes con diagnóstico psiquiátrico muestra una adecuada consistencia interna, con un coeficiente alfa de Cronbach de .86 en una versión de 17 reactivos, con tres factores y un indicador específicos para esta población. Comparada con otras validaciones del instrumento, presenta una estructura factorial similar (Alpuche et al., 2008; Galindo et al., 2015; Montero et al., 2014; Zarit et al., 1980; Zarit et al., 1985), útil para el objetivo con el que originalmente se construyó; adicionalmente, se identificó una aceptable validez concurrente teóricamente relevante con el SF-36 (Ware, 2000). Se halló que en la comparación con diferentes validaciones en poblaciones de CPI (Tabla 4) es una de las versiones con una varian- 
za explicada superior a 55.7, por lo que puede ser empleada en la atención clínica y la investigación para detectar qué CPI muestran sobrecarga, y así poder ser un instrumento específico para esta población.

Tabla 3. Correlación entre la ECZ y el SF-36.

\begin{tabular}{|l|c|c|c|c|c|}
\hline & Prom. Zarit & $\begin{array}{c}\text { Factor 1 } \\
\text { Impacto } \\
\text { de cuidado }\end{array}$ & $\begin{array}{c}\text { Factor 2 } \\
\text { Relación } \\
\text { interpersonal }\end{array}$ & $\begin{array}{c}\text { Factor 3 } \\
\text { Expectativas } \\
\text { de autoeficacia }\end{array}$ & $\begin{array}{l}\text { Indicador } \\
\text { cognitivo }\end{array}$ \\
\hline Total SF-36 & $-0.130^{*}$ & -0.121 & 0.004 & 0.022 & -0.122 \\
\hline Salud general & -0.005 & 0.047 & -0.002 & 0.012 & 0.035 \\
\hline Función física & $-0.207^{* *}$ & $-0.194^{* *}$ & $-0.179^{* *}$ & $-0.143^{*}$ & -0.054 \\
\hline Rol físico & $-0.265^{* *}$ & $-0.333^{* *}$ & $-0.154^{*}$ & $-0.132^{*}$ & -0.101 \\
\hline Rol emocional & $-0.326^{* *}$ & $-0.371^{* *}$ & $-0.170^{* *}$ & $-0.181^{* *}$ & $-0.170^{* *}$ \\
\hline Función social & 0.048 & 0.079 & 0.062 & 0.048 & 0.021 \\
\hline Dolor corporal & $0.313^{* *}$ & $0.328^{* *}$ & $0.199^{* *}$ & $0.242^{* *}$ & 0.087 \\
\hline Vitalidad & -0.024 & 0.015 & 0.060 & 0.052 & -0.075 \\
\hline Salud mental & -0.127 & $-0.163^{*}$ & 0.034 & -0.033 & -0.089 \\
\hline
\end{tabular}

**Significativa al 0.01 (bilateral).

*Significativa al 0.05 (bilateral).

Tabla 4. Comparación de diferentes validaciones del ECZ en población mexicana.

\begin{tabular}{|l|l|c|c|l|}
\hline \multicolumn{1}{|c|}{ Versiones } & \multicolumn{1}{|c|}{$\begin{array}{c}\text { Número total } \\
\text { de reactivos }\end{array}$} & $\begin{array}{c}\text { Alfa } \\
\text { de Cronbach }\end{array}$ & $\begin{array}{c}\text { Varianza } \\
\text { explicada }\end{array}$ & \multicolumn{1}{|c|}{ Factores } \\
\hline $\begin{array}{l}\text { Zarit original } \\
\text { (Zarit, Orr y Zarit, 1985). }\end{array}$ & $\begin{array}{l}1,2,3,4,5,6,7,8,9,10, \\
11,12,13,14,15,16,17, \\
18,19,20,21,22 .\end{array}$ & .83 & & $\begin{array}{l}\text { 1. Impacto del cuidado. } \\
\text { 2. Relación interpersonal. } \\
\text { 3. Expectativas de autoeficacia. }\end{array}$ \\
\hline $\begin{array}{l}\text { Alpuche et al., (2008): } \\
\text { CPI de pacientes adultos con } \\
\text { diferentes enfermedades crónicas. }\end{array}$ & $\begin{array}{l}1,2,3,4,5,6,7,8,9,10, \\
11,12,13,14,15,16,17,\end{array}$ & .90 & $50.3 \%$ & $\begin{array}{l}\text { 1. Impacto del cuidado } \\
\text { 2. Relación interpersonal. } \\
\text { 3. Expectativas de autoeficacia. }\end{array}$ \\
\hline $\begin{array}{l}\text { Montero et al., (2014): } \\
\text { CPI de niños con alguna } \\
\text { enfermedad crónica. }\end{array}$ & $\begin{array}{l}2,3,5,6,9,10,11,12,13, \\
19,20,21 .\end{array}$ & .84 & $50.0 \%$ & $\begin{array}{l}\text { 1. Impacto del cuidado. } \\
\text { 2. Relación interpersonal. } \\
\text { 3. Expectativas de autoeficacia. }\end{array}$ \\
\hline $\begin{array}{l}\text { Galindo et al., (2015): } \\
\text { CPI de pacientes adultos } \\
\text { con cáncer. }\end{array}$ & $\begin{array}{l}1,2,3,4,5,6,7,8,10,11,13,14,15,16,17,18, \\
12,13,21,22 .\end{array}$ & .90 & $51.6 \%$ & $\begin{array}{l}\text { 1. Impacto del cuidado. } \\
\text { 2. Relación interpersonal. } \\
\text { 3. Expectativas de autoeficacia. }\end{array}$ \\
\hline $\begin{array}{l}\text { Pablo, Domínguez, Hernández, } \\
\text { Salazar y Tejeda (2016): } \\
\text { CPI de pacientes con insuficiencia } \\
\text { cardiaca. }\end{array}$ & $\begin{array}{l}1,2,3,4,5,6,9,10,11,12, \\
13,16,17,18,19,22 .\end{array}$ & .90 & $51.6 \%$ & $\begin{array}{l}\text { 1. Presión. } \\
\text { 2. Carga social. }\end{array}$ \\
\hline $\begin{array}{l}\text { Flores, Rivas y Seguel (2018): } \\
\text { CPI de pacientes adultos mayores } \\
\text { con dependencia severa. }\end{array}$ & $\begin{array}{l}1,2,3,46,8,10,11,12,14, \\
15,17,18,19,20,2122 .\end{array}$ & .89 & $55.7 \%$ & $\begin{array}{l}\text { 1. Impacto del cuidado. } \\
\text { 2. Relación interpersonal. } \\
\text { 3. Expectativas de autoeficacia } \\
\text { Indicador cognitivo }\end{array}$ \\
\hline
\end{tabular}

CPI $=$ Cuidadores primarios informales .

Finalmente, según los resultados de este estudio, hubo un predominio de mujeres que fungen como cuidadores primarios $(74.9 \%)$, lo que coincide con otros trabajos que reportan porcentajes que van de 62 a 83\% (Alfaro et al., 2008; Barrón y Alvarado, 2009; Vázquez et al., 2017), lo cual implica una mayor afectación psicosocial para este grupo, ya que las mujeres en la cultura mexicana frecuentemente realizan una actividad de cuidado a diferentes miembros de la familia.

De acuerdo con Caquero, Segovia, Urritia, Miranda y Navarro (2013), cabe resaltar que la importancia de evaluar e intervenir oportunamente en los cuidadores no solamente los beneficia a 
ellos, sino que se ha documentado que el estado funcional del paciente correlaciona significativamente con dolor, vitalidad, función social, salud mental, bienestar y evaluación general de la salud del cuidador.

\section{DISCUSIÓN}

Al disponer de un instrumento específico para la población de cuidadores primarios informales de pacientes con diagnóstico psiquiátrico que cuente con la estructura psicométrica adecuada, es posible facilitar la identificación de las personas o grupos que requieran atención debido al desgaste físico, emocional y económico que genera esa tarea, que además ven afectadas sus actividades laborales, su tiempo libre y sus relaciones sociales (Montorio, Iza, López y Sánchez, 1998).

La versión de la Entrevista de Carga de Zarit para cuidadores primarios informales de pacientes con diagnostico psiquiátrico es válida y confiable, y cuenta con una estructura factorial similar a la versión original, lo que la hace más útil en la clínica y la investigación, toda vez que su aplicación puede generar programas que coadyuven a atención de la sobrecarga de esa población, toda vez que la enfermedad psiquiátrica es una enfermedad crónico-degenerativa que implica un alto nivel de dependencia del cuidado.

Los programas de intervención para los CPI de la referida población deben establecerse como un proceso de atención integrado al sistema de salud, coadyuvando así a la obtención y mantenimiento de interacciones de apoyo social, sentimientos de dominio e interacciones positivas (Vázquez et al., 2015).

Dentro de las limitaciones de este estudio están el haber contado con una muestra por conveniencia, que la población es de primer ingreso al cuadro de enfermedad y que no se genera un elevado nivel de sobrecarga, como ocurre con una persona que ya lleva muchos internamientos, cuyos cuidadores están más sobrecargados. Una perspectiva futura de investigación es que se requieren estudios longitudinales para identificar apropiadamente el nivel de sobrecarga que padecen, y que sería importante realizar un análisis confirmatorio de la estructura de la escala.

\section{REFERENCIAS}

Aguilar, J. (2014). Correlación del síndrome de desgaste y co-morbilidad psiquiátrica del cuidador primario de pacientes con esquizofrenia con el apego a la consulta externa en el Hospital Psiquiátrico Fray Bernandino Álvarez. Tesis de residencia medica de $4^{a}$ año. México: Universidad Nacional Autónoma de México.

Alfaro R., O.I., Morales V., T., Vázquez P., F., Sánchez R., S., Ramos R., B. y Guevara L., U. (2008). Sobrecarga, ansiedad y depresión en cuidadores primarios de pacientes con dolor crónico y terminales. Revista Médica del Instituto Mexicano del Seguro Social, 46,485-494.

Alpuche, V., Ramos, B., Rojas, M. y Figueroa, C. (2008). Validez de la entrevista de carga de Zarit en una muestra de cuidadores primarios informales. Psicología y Salud, 18(2), 237-245.

American Psychiatric Association (2000). Diagnostic and statistical manual of mental disorders (4 ${ }^{\text {th }}$ ed., text rev.). Washington, DC: APA.

Astudillo, A., Martínez, A., Muñoz, C., Pacheco, M. y Sepúlveda, G. (2012). Acompañamiento familiar en la hospitalización del usuario pediátrico de 6 a 12 años. Ciencia y Enfermería, 1, 67-75.

Barrón B., S. y Alvarado, S. (2009). Desgaste físico y emocional del cuidador primario en cáncer. Cancerología, 4, 39-46.

Berenzon, S., Lara, M.A., Robles, R. y Medina-Mora, M.E. (2013). Depresión: estado del conocimiento y la necesidad de políticas públicas y planes de acción en México. Salud Pública de México, 55, 74-80.

Camacho, L., Yokehed, G. y Jiménez, A. (2010). Sobrecarga del cuidador primario de personas con deterioro cognitivo y su relación con el tiempo de cuidado. Revista Enfermería Universitaria, 7(4), 35-44.

Caquero U., A., Segovia L., P., Urritia U., Ú., Miranda, C. y Navarro, E. (2013). Impacto de la relación de ayuda de cuidadores primarios en la calidad de vida de pacientes con cáncer avanzado. Psicooncología, 10, 95-108.

Flores E., G., Rivas, E. y Seguel, F. (2012). Nivel de sobrecarga en el desempeño del rol del cuidador familiar de adulto mayor con dependencia severa. Ciencia y Enfermería, 28(1), 29-41.

Galindo V., O., Benjet, C., Cruz N., H., Rojas C., E., Riveros R., A., Meneses G., A. y Alvarado A., S. (2015). Psychometric properties of the Zarit Burden Interview in Mexican caregivers of cancer patients. Psycho-Oncology, 24(5), 612-615. doi: 10.1002/pon.3686. 
Islas N., L., Ramos R., B., Aguilar, M. y García, M. (2006). Perfil psicosocial del cuidador primario informal del paciente con EPOC. Revista del Instituto Nacional de Enfermedades Respiratorias, 19(4), 266-271.

Kessler, R.C., Angermeyer, M., Anthony, J.C., De Graaf, R., Demyttenaere, K. y Gasquet, I. (2007). Lifetime prevalence and age-of-onset distributions of mental disorders in the World Health Organization's World Mental Health Survey Initiative. World Psychiatry, 6, 168-176.

Lara, C., Medina-Mora, M,E., Borges, G. y Zambrano, J. (2007). Social cost of mental disorders: disability and work days lost. Results from the Mexican Survey of Psychiatric Epidemiology. Salud Mental, 30(5), 4-11.

Montorio, C., Iza, F., López, L. y Sánchez, C. (1998). La entrevista de carga del cuidador primario. Utilidad y validez del concepto de carga. Anales de Psicología, 14(2), 229-248.

Montero, P., Jurado, S., Valencia, A., Méndez, J. y Mora, I. (2014). Escala de Carga del Cuidador de Zarit: evidencia de validez en México. Psicooncología, 11(1), 71-85. doi: 10.5209/rev_PSIC. 2014.v11.n1.44918.

Murray, C.J., Vos, T., Lozano, R., Naghavi, M., Flaxman, A.D., Michaud, C. y Aboyans, V. (2012). Disability-adjusted life years (DALYs) for 291 diseases and injuries in 21 regions, 1990-2010: A systematic analysis for the Global Burden of Disease Study 2010. The Lancet, 380(9859), 2197-2223.

Secretaría de Salud (2006). Programa de acción: Salud Mental 2006. México: Autor.

Organización Mundial de la Salud (2004). Invertir en salud mental. Ginebra: OMS.

Pablo R., S., Domínguez B., T., Hernández V., P., Salazar S., R. y Tejeda A., O. (2016). Propiedades psicométricas de la Escala de Carga de Zarit para cuidadores de pacientes con insuficiencia cardíaca. Integración Académica en Psicología, 4(11), 93-100.

Vázquez, O., Castillo, E., Huertas, L., García, A., Ponce, J.L., Manzanilla, E. y Aguilar, S.A. (2015). Guía de práctica clínica para la atención psico-oncológica del cuidador primario informal de pacientes con cáncer. Psicooncología, 12(1), 87-104. doi: 10.5209/rev_PSIC. 2015.v12.n1.48906.

Vázquez O., G., García A., M., Gómez Á., H., Galeana P., C., Bernal L., S., Rivera F., L. y Ponce J., L.A. (2017). Propiedades psicométricas del Cuestionario General de la Salud de Goldberd (QHQ-28) en cuidadores primarios informales de pacientes con cáncer. Psicooncología, 14(1), 71.

Ware, E. (2000). SF-36 Health Survey Update. Spine, 24, 3130-3139.

Zarit, S., Reever, E. y Bach-Peterson, J. (1980). Relatives of the impaired elderly: correlates of feelings burden. The Gerontologist, 20(6), 649-655. doi: 10.1093/geront/20.6.649.

Zarit, S., Orr, N. y Zarit, J. (1985). The hidden victims of Alzheimer's disease: Families under stress. New York: New York University Press. 